\title{
Correlating Complementary Data for Improving Electron Backscatter Diffraction (EBSD) Microstructural Characterization of Geological Materials
} \author{
I. Wright ${ }^{1}$ \\ 1. EDAX, A Division of Ametek, Materials Analysis Division, Draper UT USA \\ 2. EDAX, A Division of Ametek, Materials Analysis Division, Mahwah NJ USA \\ 3. EDAX B.V., Tilburg The Netherlands
}

Matthew M. Nowell ${ }^{1}$, Shawn W. Wallace ${ }^{2}$, Jens Rafaelsen ${ }^{2}$, Tara L. Nylese ${ }^{2}$, René de Kloe ${ }^{3}$, and Stuart

Electron Backscattered Diffraction (EBSD) has become widely accepted for the crystallographic microstructural characterization of geological materials [1]. For comprehensive multi-phase material analysis, correlating the EBSD structural data with complementary compositional information from analytical techniques including Energy Dispersive Spectroscopy (EDS), Wavelength Dispersive Spectroscopy (WDS), and Cathodoluminescence (CL) can improve EBSD phase differentiation performance and provides a more complete description of each analyzed phase.

When an EDS detector is properly positioned relative to a highly-tilted EBSD sample, EDS data can be collected simultaneously with the EBSD data. While the EDS and EBSD interaction volumes are not exactly coincident, the spatial mismatch is small $(<\approx 1 \mu \mathrm{m})$ and the difference can generally be ignored when the mapping step size and features of interest are larger than this mismatch. In these cases, the EDS and EBSD data can be considered directly correlated. The EDS data can then be used to assist EBSD phase differentiation for structurally similar phases, as shown in Figure 1. In this example, an exsolved sample of Ilmenite and Hematite was analyzed. This sample comes from the Saint Urbain Anorthosite Massif, a complex magmatic system, aged $\sim 1 \mathrm{Ga}$ [2], that contains economic deposits of Fe and Ti. The exsolution of the Ilmenite and Hematite is due to extremely slow cooling rates $\left(\sim 3^{\circ} \mathrm{C} /\right.$ million years) [2] of the residual $\mathrm{Ti}$ and $\mathrm{Fe}$ rich liquid. Both phases have a trigonal crystal structure with similar lattice parameters, so it is difficult for EBSD to consistently and reliably differentiate these phases (Figure 1a). However, the EDS information easily differentiates these phases. By using the correlated EDS information to select the correct phase for EBSD analysis, more accurate phase and microstructural characterization is obtained (Figure 1b) [3].

When the step size and features of interest are smaller than the spatial mismatch of the EDS and EBSD interaction volumes, direct correlation can no longer be assumed. While Fig. 1 shows large scale exsolution, some exsolved grains are smaller than/equal to the EDS interaction volume used at normal EBSD operating conditions (15KV and greater). Due to this change in scale, the optimal operating conditions for EBSD data collection and EDS are much different (Figure 2), either requiring some give and take in one of the methods or collecting the data at different times and/or operation conditions. With this in mind, it may be desirable to collect EDS, or other compositional and/or complementary information (WDS, CL, EBIC, etc) in a traditional, non-tilted geometry. This could be done on the same instrument, or also on another SEM or microprobe. In these cases, a routine has been implemented that allows a set of images to be spatially correlated with the EBSD mapping data by selecting 6 or more coincident points within the shared field of analysis. A displacement vector field is then calculated for and applied to the image set, and the subsequent correlated images is then available for subsequent analysis. This approach allows for other analytical techniques to be used for phase differentiation problems or for correlating with specific microstructural features. 


\section{References:}

[1] D.J. Prior, E. Mariani, and J. Wheeler in "Electron Backscatter Diffraction in Materials Science $2^{\text {nd }}$ Edition", ed. A. J. Schwartz, M. Kumar, B.L. Adams, and D.P. Field, (Springer, New York) p. 345.

[2] C.E. Morisset, et. al, Precambrian Research 174 (2009), p. 95.

[3] M.M. Nowell and S.I. Wright, Journal of Microscopy 213 (2004), p.296.

[4] D. Drouin et al, SCANNING 29 (2007), p. 92.

[5] DTSA2: http://www.cstl.nist.gov/div837/837.02/epq/dtsa2/
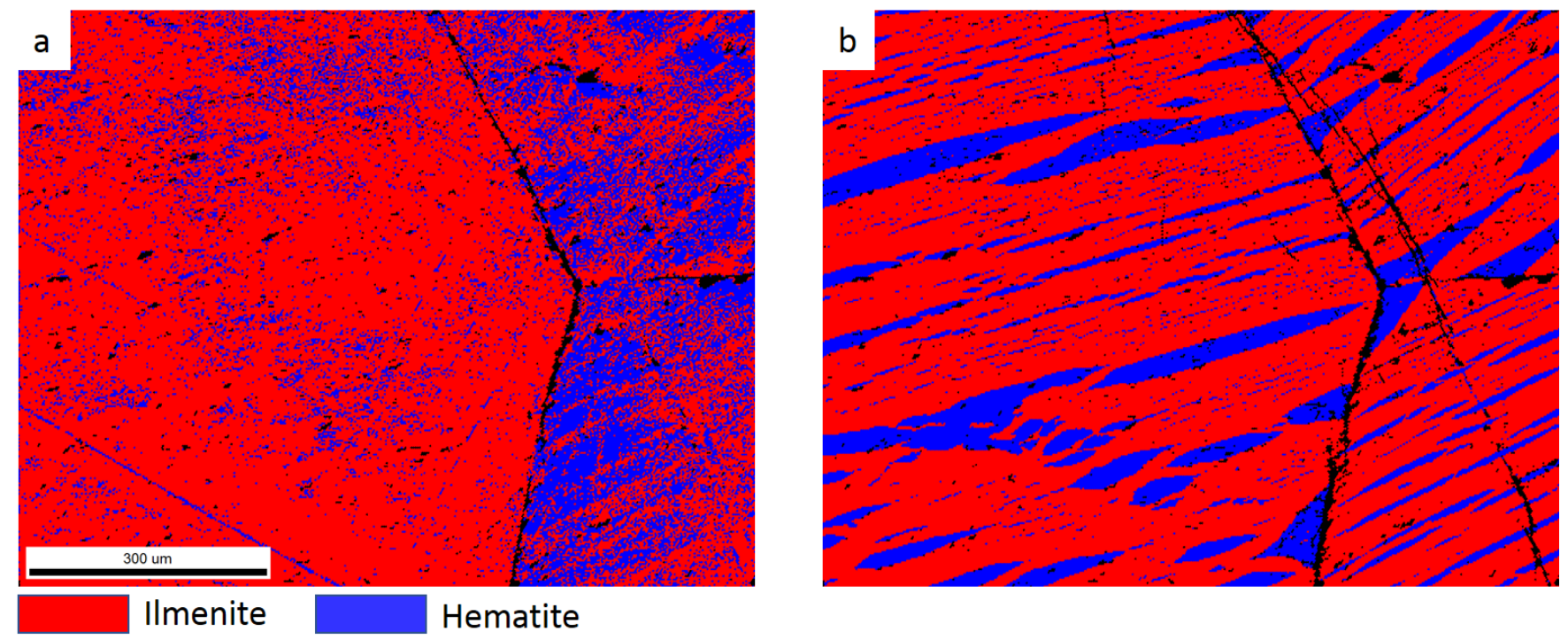

Figure 1. Phase map of Ilmenite and Hematite exsolution microstructure using a) EBSD only and b) correlated EDS and EBSD information for phase differentiation.
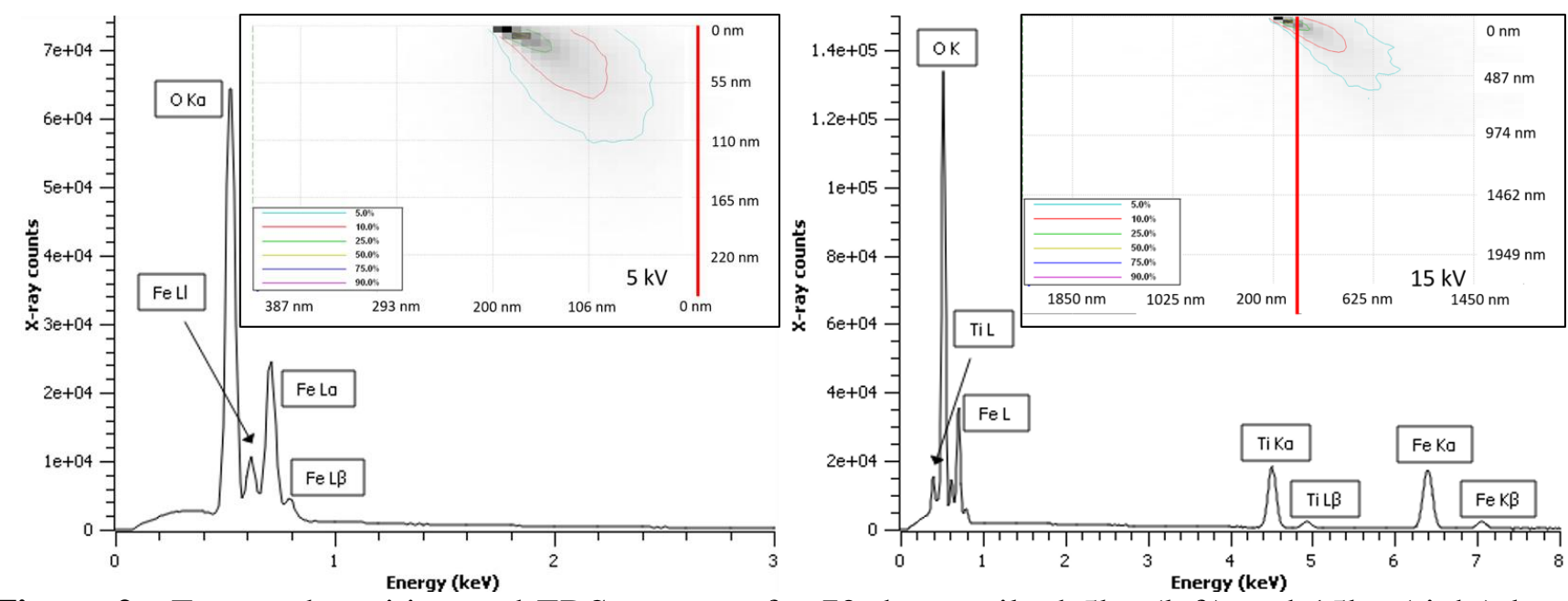

Figure 2. Energy deposition and EDS spectra of a 70 degree tilted $5 \mathrm{kv}$ (left) and $15 \mathrm{kv}$ (right) beam landing $200 \mathrm{~nm}$ away from an Hematite-Ilmenite Grain Boundary (red line). The energy deposition was simulated using the Casino software package [4] while the spectra were simulated using DTSA-II[5]. For a $5 \mathrm{kv}$ beam, the bulk of the energy is deposited very near the intersection point but still within the Hematite grain and no Ti signal is seen in the EDS spectrum. For the $15 \mathrm{kv}$ beam, a typical EBSD value, the energy is deposited across a grain boundary, generation EDS signal from both phases. 